\title{
Erratum: Centroid theory of transverse electron-proton two-stream instability in a long proton bunch [Phys. Rev. ST Accel. Beams 6, 014204 (2003)]
}

\author{
Tai-Sen F. Wang, Paul J. Channell, Robert J. Macek, and Ronald C. Davidson
}

(Received 27 April 2004; published 1 June 2004)

We have identified a few algebraic errors in our recent paper [1]. Although the overall qualitative results in the original paper are still correct, an error in Eq. (42) does cause some minor changes in the quantitative results and alternations in many equations. The most significant change is in the required frequency spread for stability given in Eq. (68), which is now found to be twice that given in the original paper [see Eq. (68) below]. The details of the revisions are listed in the following.

First, the following four sentences should be inserted after Eq. (12) to comment on the procedure of obtaining the centroid equations and the applicability of Eq. (12) in the case of nonuniform proton line density: "Using the same procedure and the full solutions of Eqs. (3) and (4) one can derive the identical results in Eqs. (11) and (12). Note that Eq. (6) and the procedure of deriving Eq. (12) are valid for uniform $\lambda_{p}$. For nonuniform proton line density, the $\omega_{e}^{2}$ in Eq. (4) is replaced by a function proportional to $\lambda_{p}$, and $y_{e}=A(t) \exp \left[i \int \omega_{e}(t) d t\right]+A^{*}(t) \exp \left[-i \int \omega_{e}(t) d t\right]$. Equation (12) should be a good approximation for describing the motion of the electron centroid when $\Delta_{e} \ll \omega_{e o}$ and $\left|A^{-1} d A / d t\right| \ll \omega_{e} . "$

Next, in Eqs. (34), (36), and (38), the equal sign $(=)$ should be replaced by the approximate sign $(\approx)$ to indicate the omission of the initial conditions; i.e., these equations should read

$$
\begin{gathered}
\tilde{Y}\left(z^{\prime}, \omega\right) \approx \frac{G\left(z^{\prime}\right) e^{\left(\Delta_{p}-\Delta_{e}-i \omega\right) z^{\prime} / v}}{v^{2} W\left(\omega_{\beta}^{2}-\omega^{2}\right)} \int_{0}^{z^{\prime}} \Omega^{2}(x)\left[\Phi\left(z^{\prime}\right) \Psi(x)-\Psi\left(z^{\prime}\right) \Phi(x)\right] \tilde{Y}(x, \omega) e^{-\left(\Delta_{p}-\Delta_{e}-i \omega\right) x / v} d x, \\
\hat{Y}\left(z^{\prime}, \omega\right) \approx \frac{1}{v^{2} W\left(\omega_{\beta}^{2}-\omega^{2}\right)} \int_{0}^{z^{\prime}} \Omega^{2}(x) G(x)\left[\Phi\left(z^{\prime}\right) \Psi(x)-\Psi\left(z^{\prime}\right) \Phi(x)\right] \hat{Y}(x, \omega) d x,
\end{gathered}
$$

and

$$
\zeta\left(z^{\prime}, \omega\right) \approx \frac{1}{v^{2} W\left(\omega_{\beta}^{2}-\omega^{2}\right)} \int_{0}^{z^{\prime}} \Omega^{2}(x) G(x) \Phi(x) \Psi(x)\left[1-\frac{\Psi\left(z^{\prime}\right) \Phi(x)}{\Phi\left(z^{\prime}\right) \Psi(x)}\right] \zeta(x, \omega) d x .
$$

The most consequential revision is in Eq. (42). The solution given in Eq. (42) should read

$$
\zeta\left(z^{\prime}, \omega\right) \approx\left(\frac{1}{\omega^{2}-\omega_{\beta}^{2}}\right) \exp \left[\frac{i \omega_{\beta}^{2} J\left(z^{\prime}\right)}{\omega^{2}-\omega_{\beta}^{2}}\right],
$$

where the factor $1 /\left(\omega^{2}-\omega_{\beta}^{2}\right)$ before the exponential function is needed for the inverse Fourier transformation to yield the correct betatron-oscillation solution for $Y_{p}$. Accordingly, the normalization factors in Eqs. (37) and (48) should be changed from $\omega_{\beta}^{-3}$ to $\omega_{\beta}^{-1}$ in order to make the dimension of $Y_{p}$ the same as $\Phi$ and $\Psi$. Therefore

$$
\hat{Y}\left(z^{\prime}, \omega\right)=\omega_{\beta}^{-1} \zeta\left(z^{\prime}, \omega\right) \Phi\left(z^{\prime}\right)
$$

and

$$
\hat{Y}\left(z^{\prime}, \omega\right)=\omega_{\beta}^{-1} \zeta\left(z^{\prime}, \omega\right) \Psi\left(z^{\prime}\right) .
$$

The corrections in Eqs. (37), (42), and (48) lead to the following revisions in Eqs. (43), (45), (46), (49)-(62), (65), (66), (68), (73), (77), and (78):

$$
\begin{gathered}
\tilde{Y}\left(z^{\prime}, \omega\right) \approx \frac{\Phi\left(z^{\prime}\right) G\left(z^{\prime}\right)}{\omega_{\beta}\left(\omega^{2}-\omega_{\beta}^{2}\right)} \exp \left[\left(\Delta_{p}-\Delta_{e}-i \omega\right) \frac{z^{\prime}}{v}+\frac{i \omega_{\beta}^{2} J\left(z^{\prime}\right)}{\omega^{2}-\omega_{\beta}^{2}}\right], \\
Y_{p}\left(z^{\prime}, t\right) \sim \frac{G\left(z^{\prime}\right)}{\omega_{\beta} \sqrt{2 \pi}} \Phi\left(z^{\prime}\right) e^{-\Delta_{p}\left(t-z^{\prime} / v\right)} e^{-\Delta_{e} z^{\prime} / v} \int_{-\infty}^{\infty}\left(\frac{1}{\omega^{2}-\omega_{\beta}^{2}}\right) \exp \left[i \omega\left(t-z^{\prime} / v\right)+\frac{i \omega_{\beta}^{2} J\left(z^{\prime}\right)}{\omega^{2}-\omega_{\beta}^{2}}\right] d \omega,
\end{gathered}
$$




$$
\begin{aligned}
& Y_{p}\left(z^{\prime}, t\right) \sim-i \sqrt{\frac{\pi}{2}} \xi\left(z^{\prime}\right) \Phi\left(z^{\prime}\right) \exp \left[-\Delta_{p}\left(t-\frac{z^{\prime}}{v}\right)-\Delta_{e}\left(\frac{z^{\prime}}{v}\right)-\frac{i J\left(z^{\prime}\right)}{4}\right] \\
& \times\left\{e^{-i \omega_{\beta}\left(t-z^{\prime} / v\right)}\left[I_{0}(u)-\frac{i J\left(z^{\prime}\right) I_{1}(u)}{2 u}\right]-e^{i \omega_{\beta}\left(t-z^{\prime} / v\right)}\left[J_{0}(u)-\frac{i J\left(z^{\prime}\right) J_{1}(u)}{2 u}\right]\right\}, \\
& Y_{p}\left(z^{\prime}, t\right) \sim i \sqrt{\frac{\pi}{2}} \xi\left(z^{\prime}\right) \Psi\left(z^{\prime}\right) \exp \left[-\Delta_{p}\left(t-\frac{z^{\prime}}{v}\right)-\Delta_{e}\left(\frac{z^{\prime}}{v}\right)+\frac{i J\left(z^{\prime}\right)}{4}\right] \\
& \times\left\{e^{i \omega_{\beta}\left(t-z^{\prime} / v\right)}\left[I_{0}(u)+\frac{i J\left(z^{\prime}\right) I_{1}(u)}{2 u}\right]-e^{-i \omega_{\beta}\left(t-z^{\prime} / v\right)}\left[J_{0}(u)+\frac{i J\left(z^{\prime}\right) J_{1}(u)}{2 u}\right]\right\}, \\
& Y_{p}\left(z^{\prime}, t\right) \approx C_{p} M_{p}\left(z^{\prime}\right) e^{-\Delta_{p} t}\left\{\left[I_{0}^{2}(u)+\frac{J^{2}\left(z^{\prime}\right) I_{1}^{2}(u)}{4 u^{2}}\right]^{1 / 2} \cos T_{p}-\left[J_{0}^{2}(u)+\frac{J^{2}\left(z^{\prime}\right) J_{1}^{2}(u)}{4 u^{2}}\right]^{1 / 2} \cos S_{p}\right\}, \\
& M_{p}\left(z^{\prime}\right)=\xi\left(z^{\prime}\right) R\left(z^{\prime}\right) \exp \left[\left(\Delta_{p}-\Delta_{e}\right) z^{\prime} / v\right] \\
& T_{p}=T_{p}\left(z^{\prime}, t\right)=\sigma_{p}+\psi_{p}-\omega_{\beta}\left(t-z^{\prime} / v\right)+\Theta\left(z^{\prime}\right)-J\left(z^{\prime}\right) / 4 \\
& S_{p}=S_{p}\left(z^{\prime}, t\right)=\sigma_{p}+\phi_{p}+\omega_{\beta}\left(t-z^{\prime} / v\right)+\Theta\left(z^{\prime}\right)-J\left(z^{\prime}\right) / 4 \\
& Y_{p}(z, t) \sim-i \sqrt{\frac{\pi}{2}} \xi(z+v t) \Phi(z+v t) \exp \left[-\Delta_{p}\left(\frac{z}{v}\right)-\Delta_{e}\left(t+\frac{z}{v}\right)-\frac{i J(z+v t)}{4}\right] \\
& \times\left\{e^{-i \omega_{\beta} z / v}\left[I_{0}(\tilde{u})-\frac{i J(z+v t) I_{1}(\tilde{u})}{2 \tilde{u}}\right]-e^{i \omega_{\beta} z / v}\left[J_{0}(\tilde{u})-\frac{i J(z+v t) J_{1}(\tilde{u})}{2 \tilde{u}}\right]\right\}, \\
& Y_{p}\left(z^{\prime}, t\right) \approx C_{p} M_{p}\left(z^{\prime}\right) e^{-\Delta_{p} t}\left[I_{0}^{2}(u)+\frac{J^{2}\left(z^{\prime}\right) I_{1}^{2}(u)}{4 u^{2}}\right]^{1 / 2} \cos T_{p},
\end{aligned}
$$

$$
\begin{aligned}
Y_{p}\left(z^{\prime}, t\right) \approx \frac{C_{p}^{\prime} \xi\left(z^{\prime}\right) R\left(z^{\prime}\right)}{\left[2 \omega_{\beta} J\left(z^{\prime}\right)\left(t-z^{\prime} / v\right)\right]^{1 / 4}} \cos \left[\sigma_{p}+\Theta\left(z^{\prime}\right)-\omega_{\beta}\left(t-z^{\prime} / v\right)-J\left(z^{\prime}\right) / 4\right] \exp [ & -\Delta_{p}\left(t-\frac{z^{\prime}}{v}\right)-\Delta_{e}\left(\frac{z^{\prime}}{v}\right) \\
& \left.+\sqrt{2 \omega_{\beta} J\left(z^{\prime}\right)\left(t-z^{\prime} / v\right)}\right],
\end{aligned}
$$

$$
\begin{gathered}
Y_{e}\left(z^{\prime}, t\right) \sim \sqrt{2 \pi} J\left(z^{\prime}\right) \Phi\left(z^{\prime}\right) \exp \left[-\frac{i J\left(z^{\prime}\right)}{4}-\Delta_{p}\left(t-\frac{z^{\prime}}{v}\right)-\Delta_{e}\left(\frac{z^{\prime}}{v}\right)\right] \\
\times\left\{e^{-i \omega_{\beta}\left(t-z^{\prime} / v\right)}\left[\frac{I_{1}(u)}{u}-\frac{J^{2}\left(z^{\prime}\right) I_{2}(u)}{8 u^{2}}\right]-e^{i \omega_{\beta}\left(t-z^{\prime} / v\right)}\left[\frac{J_{1}(u)}{u}-\frac{J^{2}\left(z^{\prime}\right) J_{2}(u)}{8 u^{2}}\right]\right\} \\
Y_{e}\left(z^{\prime}, t\right) \approx C_{e} M_{e}\left(z^{\prime}\right) e^{-\Delta_{p} t}\left[\frac{I_{1}(u)}{u}-\frac{J^{2}\left(z^{\prime}\right) I_{2}(u)}{8 u^{2}}\right] \cos T_{e} \\
M_{e}\left(z^{\prime}\right)=J\left(z^{\prime}\right) R\left(z^{\prime}\right) \exp \left[\left(\Delta_{p}-\Delta_{e}\right) z^{\prime} / v\right] \\
T_{e}=T_{e}\left(z^{\prime}, t\right)=\psi_{e}+\sigma_{e}-\omega_{\beta}\left(t-z^{\prime} / v\right)+\Theta\left(z^{\prime}\right)-J\left(z^{\prime}\right) / 4 \\
\psi_{e}=0
\end{gathered}
$$

$$
\begin{aligned}
Y_{e}\left(z^{\prime}, t\right) \approx \frac{C_{e}^{\prime} J\left(z^{\prime}\right) R\left(z^{\prime}\right)}{\left[2 \omega_{\beta} J\left(z^{\prime}\right)\left(t-z^{\prime} / v\right)\right]^{3 / 4}} \cos \left[\sigma_{e}+\Theta\left(z^{\prime}\right)-\omega_{\beta}\left(t-z^{\prime} / v\right)-J\left(z^{\prime}\right) / 4\right] \exp [ & -\Delta_{p}\left(t-\frac{z^{\prime}}{v}\right)-\Delta_{e}\left(\frac{z^{\prime}}{v}\right) \\
& \left.+\sqrt{2 \omega_{\beta} J\left(z^{\prime}\right)\left(t-z^{\prime} / v\right)}\right]
\end{aligned}
$$




$$
\begin{gathered}
\Gamma_{p}\left(z^{\prime}, t\right) \approx-\Delta_{p}+\frac{\omega_{\beta} J\left(z^{\prime}\right) I_{1}(u)\left[4 u^{2} I_{0}(u)+J^{2}\left(z^{\prime}\right) I_{2}(u)\right]}{u\left[4 u^{2} I_{0}^{2}(u)+J^{2}\left(z^{\prime}\right) I_{1}^{2}(u)\right]}, \\
\Gamma_{e}\left(z^{\prime}, t\right) \approx-\Delta_{p}+\frac{\omega_{\beta} J\left(z^{\prime}\right)\left[8 u I_{2}(u)-J^{2}\left(z^{\prime}\right) I_{3}(u)\right]}{u\left[8 u I_{1}(u)-J^{2}\left(z^{\prime}\right) I_{2}(u)\right]}, \\
\left(\frac{\Delta_{p}}{\omega_{\beta}}\right)_{t} \approx \max \left\{\frac{J\left(z^{\prime}\right)}{2}\left[\frac{1+J^{2}\left(z^{\prime}\right) / 32}{1+J^{2}\left(z^{\prime}\right) / 16}\right]\right\}, \\
\tilde{Y}_{p}\left(z^{\prime}, \omega\right) \approx G \hat{Y}\left(z^{\prime}, \omega-i \Delta_{p}\right) e^{-i\left(\omega-i \Delta_{e}\right) z^{\prime} / v}=\frac{G \Phi\left(z^{\prime}\right)}{\omega_{\beta}\left(\omega^{2}-\omega_{\beta}^{2}\right)} \exp \left[-i\left(\omega-i \Delta_{e}\right)\left(\frac{z^{\prime}}{v}\right)+\frac{i \omega_{\beta}^{2} J\left(z^{\prime}\right)}{\left(\omega-i \Delta_{p}\right)^{2}-\omega_{\beta}^{2}}\right], \\
Y_{p}\left(z^{\prime}, t\right) \approx C e^{-\Delta_{p} t+\left(\Delta_{p}-\Delta_{e}\right) z^{\prime} / v}\left\{\left[I_{0}^{2}(u)+\frac{J^{2}\left(z^{\prime}\right) I_{1}^{2}(u)}{4 u^{2}}\right]^{1 / 2} \cos T-\left[J_{0}^{2}(u)+\frac{J^{2}\left(z^{\prime}\right) J_{1}^{2}(u)}{4 u^{2}}\right]^{1 / 2} \cos S\right\},
\end{gathered}
$$

and

$$
\begin{aligned}
Y_{p}\left(z^{\prime}, t\right) \approx \frac{\hat{C}}{\left[\omega_{0} \omega_{\beta}\left(z^{\prime} / v\right)\left(t-z^{\prime} / v\right)\right]^{1 / 4}} \cos \left[\sigma+\left(\omega_{0}+\omega_{\beta}-\xi \omega_{0} / 8\right)\left(z^{\prime} / v\right)-\omega_{\beta} t\right] \exp [ & -\Delta_{p}\left(t-\frac{z^{\prime}}{v}\right)-\Delta_{e}\left(\frac{z^{\prime}}{v}\right) \\
& +\sqrt{\left.\xi \omega_{0} \omega_{\beta}\left(\frac{z^{\prime}}{v}\right)\left(t-\frac{z^{\prime}}{v}\right)\right] .}
\end{aligned}
$$

In Eq. (53), $\psi_{p}=\cos ^{-1}\left\{2 u I_{0}(u)\left[4 u^{2} I_{0}^{2}(u)+J^{2}\left(z^{\prime}\right) I_{1}^{2}(u)\right]^{-1 / 2}\right\}$, and $\phi_{p}=\cos ^{-1}\left\{2 u J_{0}(u)\left[4 u^{2} J_{0}^{2}(u)+J^{2}\left(z^{\prime}\right) J_{1}^{2}(u)\right]^{-1 / 2}\right\}$. The solution given in Eq. (46) is the same as that obtained from retaining the $n=0, m=0$, and $m=1$ terms in the exact solution given in Eq. (A13) in Appendix A. The approximate solution for $Y_{e}\left(z^{\prime}, t\right)$ to lowest order in $J\left(z^{\prime}\right) / u$ shown in Eq. (57) is obtained by retaining the $n=0, m=1 \mathrm{~m}$ and $m=2$ terms in Eq. (A17). Note that the growth rate given in Eq. (68) is twice as large as that given in the original paper. As a result, the maximum of $J\left(z^{\prime}\right)$ presented in the numerical example is about 0.057 , and therefore according to Eq. (68), the instability threshold is near $\left(\Delta_{p} / \omega_{\beta}\right)_{t} \approx$ $2.9 \%$ instead of $1.4 \%$ obtained previously [1].

Because of the revisions in Eqs. (55), (58), (65), and (67), Figs. 3-5 in the original paper have to be corrected according to the following (there are no changes to Figs. 1 and 2):

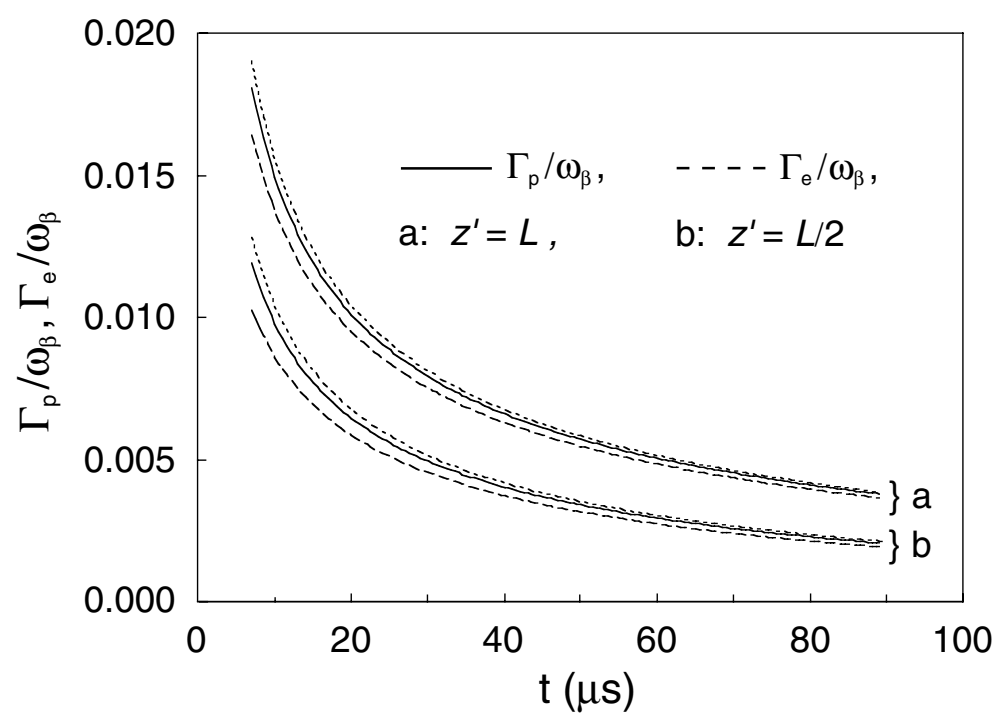

FIG. 3. The growth rates at the tail $\left(z^{\prime}=L\right)$ and the center $\left(z^{\prime}=L / 2\right)$ of the proton bunch are shown as functions of time. Here, $\Gamma_{p}$ and $\Gamma_{e}$ have been normalized to the betatron frequency $\omega_{\beta}$. The solid curve and the dashed curve are computed according to Eqs. (65) and (66), respectively. The dotted curve is computed using the asymptotic approximation in Eq. (67). The parameter values are described in the text. 


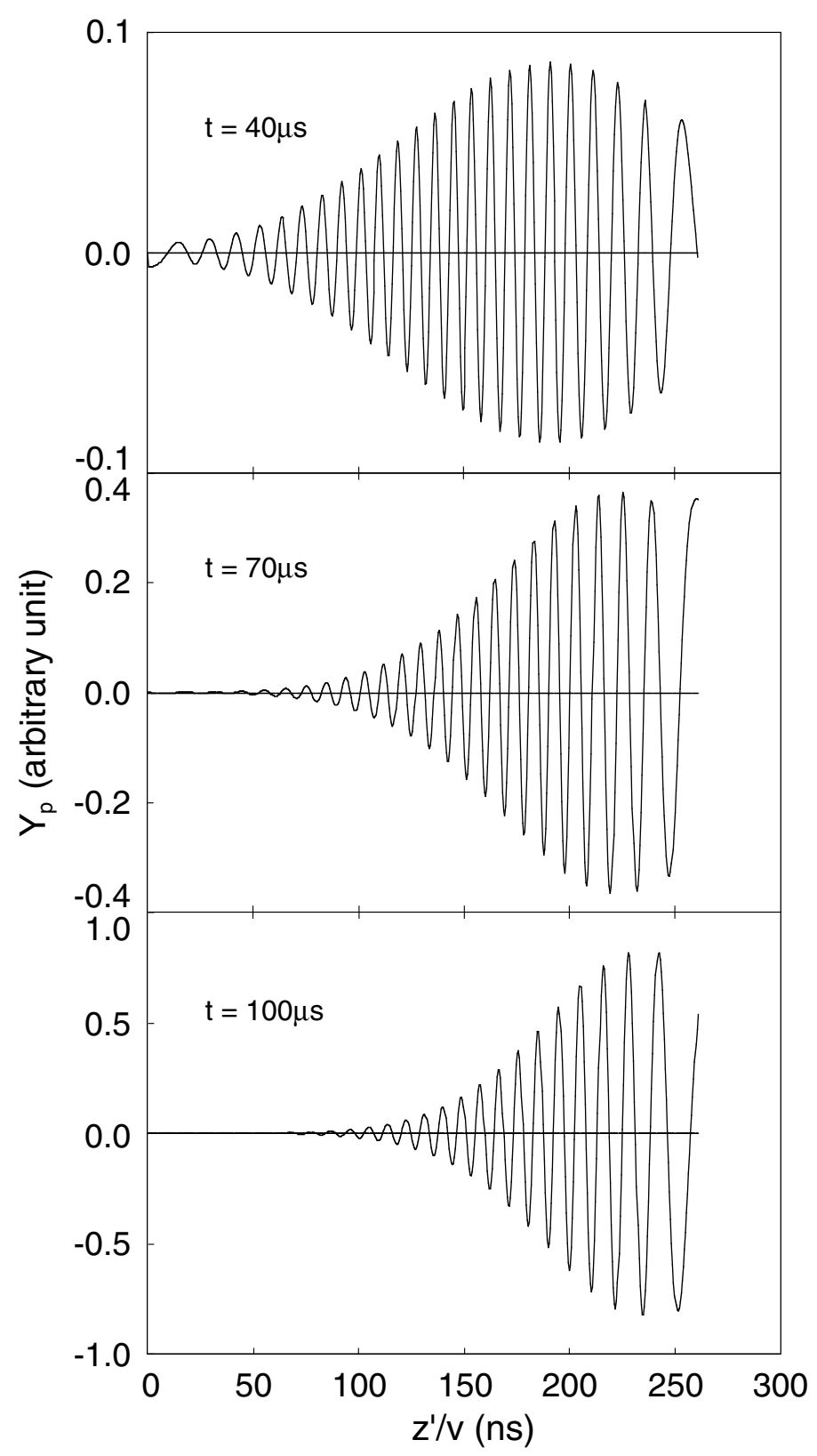

FIG. 4. The growing portion of the perturbed proton motion $Y_{p}$ as a function of $z^{\prime} / v$ at time $t=40,70$, and $100 \mu$ s. Here, the value of $Y_{p}$ is computed using Eq. (55) with $\sigma_{p}=0$ and the parameter values described in the text. The proton bunch is $260 \mathrm{~ns}$ long. The vertical axis has arbitrary units.

The corrections in Eqs. (37), (42), and (48) also cause some changes in Eqs. (A1), (A3), (A11)-(A13), (A16), and (A17) in Appendix A. These equations should read

$$
\begin{gathered}
\hat{Y}\left(z^{\prime}, \omega\right) \approx \frac{\Phi\left(z^{\prime}\right)}{\omega_{\beta}\left(\omega^{2}-\omega_{\beta}^{2}\right)} \exp \left[\frac{i \omega_{\beta}^{2} J\left(z^{\prime}\right)}{\omega^{2}-\omega_{\beta}^{2}}\right], \\
\hat{Y}\left(z^{\prime}, \omega\right)=\frac{Q\left(z^{\prime}, \omega\right) \Phi\left(z^{\prime}\right)}{\omega_{\beta}\left(\omega^{2}-\omega_{\beta}^{2}\right)} \exp \left[\frac{i \omega_{\beta}^{2} J\left(z^{\prime}\right)}{\omega^{2}-\omega_{\beta}^{2}}\right], \\
\hat{Y}\left(z^{\prime}, \omega\right)=\Phi\left(z^{\prime}\right) \exp \left[\frac{i \omega_{\beta}^{2} J\left(z^{\prime}\right)}{\omega^{2}-\omega_{\beta}^{2}}\right] \sum_{n=0}^{\infty} \frac{a_{n}\left(z^{\prime}\right)}{\omega_{\beta}\left(\omega^{2}-\omega_{\beta}^{2}\right)^{n+1}},
\end{gathered}
$$




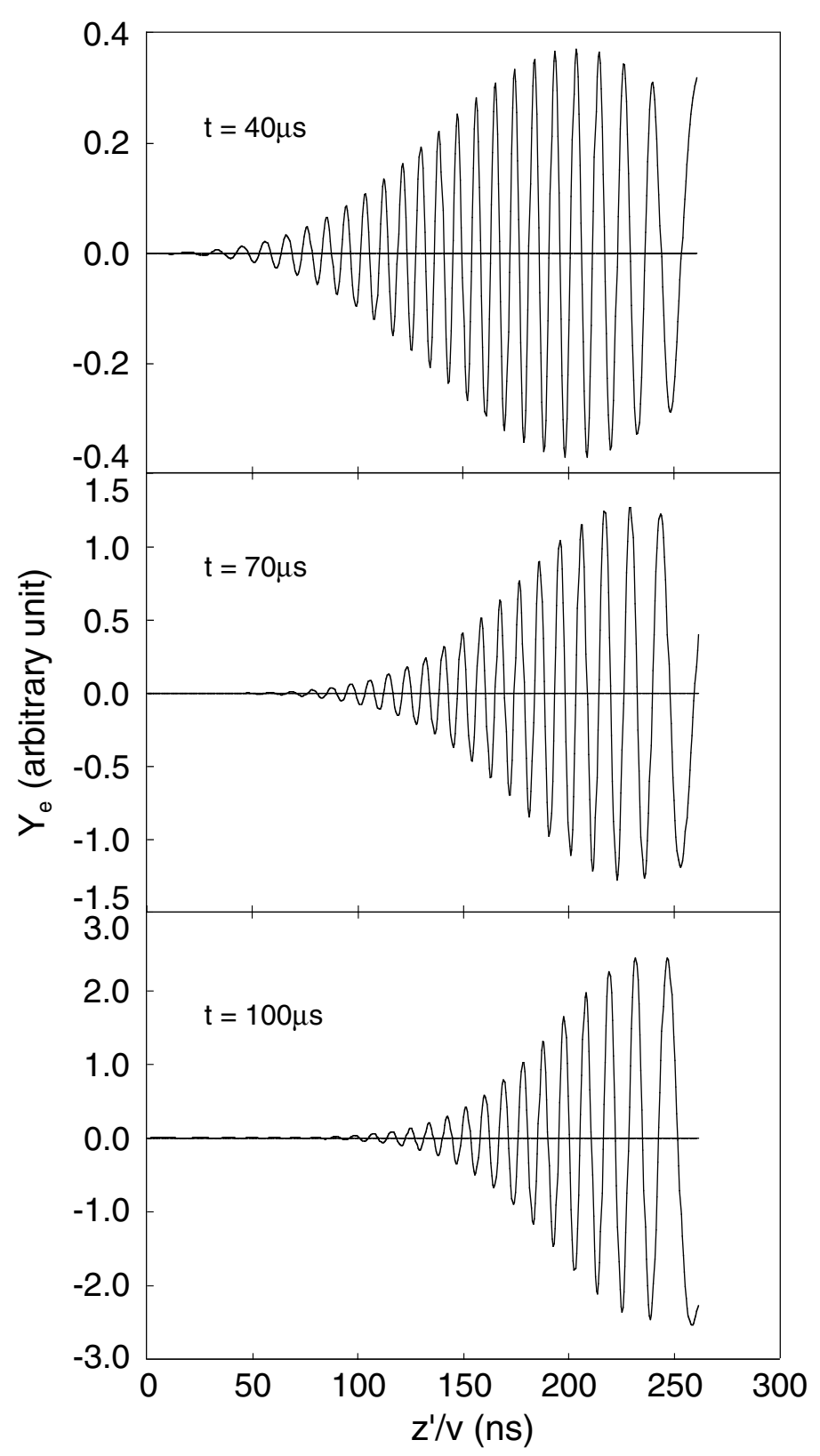

FIG. 5. The growing portion of the perturbed electron motion $Y_{e}$ as a function of $z^{\prime} / v$ at time $t=40,70$, and $100 \mu$ s. Here, the value of $Y_{e}$ is computed using Eq. (58) with $\sigma_{e}=\pi / 2$ and the parameter values described in the text. The proton bunch is $260 \mathrm{~ns}$ long. The vertical axis has arbitrary units.

$$
\begin{gathered}
Y_{p}\left(z^{\prime}, t\right)=\frac{\omega_{\beta}}{\sqrt{2 \pi}} \xi\left(z^{\prime}\right) \Phi\left(z^{\prime}\right) e^{-\Delta_{p}\left(t-z^{\prime} / v\right)-\Delta_{e}\left(z^{\prime} / v\right)} \sum_{n=0}^{\infty} a_{n} \vartheta_{n+1}, \\
Y_{p}\left(z^{\prime}, t\right)=-i \sqrt{\frac{\pi}{2}} \xi\left(z^{\prime}\right) \Phi\left(z^{\prime}\right) e^{-\Delta_{p}\left(t-z^{\prime} / v\right)-\Delta_{e}\left(z^{\prime} / v\right)} \sum_{n=0}^{\infty} \frac{a_{n}}{\left(2 \omega_{\beta}\right)^{2 n}} \sum_{m=-n}^{\infty} \frac{1}{(m+n) !}\left(\frac{i u}{4 \alpha}\right)^{m} \\
\times\left[e^{-i \alpha} I_{m}(u)-e^{i \alpha} J_{m}(u)\right] \frac{d^{m+n}}{d p^{m+n}}\left[\left.\frac{1}{p^{n+1}} \exp \left(\frac{-i u^{2}}{8 \alpha p}\right)\right|_{p=1},\right. \\
Y_{e}\left(z^{\prime}, t\right)=\frac{-1}{\sqrt{2 \pi}} \Phi\left(z^{\prime}\right) e^{-\Delta_{p}\left(t-z^{\prime} / v\right)-\Delta_{e}\left(z^{\prime} / v\right)} \sum_{n=0}^{\infty} \frac{a_{n}}{\omega_{\beta}} \vartheta_{n},
\end{gathered}
$$


and

$$
\begin{aligned}
Y_{e}\left(z^{\prime}, t\right)= & i 2 \sqrt{2 \pi} \Phi\left(z^{\prime}\right) e^{-\Delta_{p}\left(t-z^{\prime} / v\right)-\Delta_{e}\left(z^{\prime} / v\right)} \sum_{n=0}^{\infty} \frac{a_{n}}{\left(2 \omega_{\beta}\right)^{2 n}} \sum_{m=1-n}^{\infty} \frac{1}{(m+n-1) !}\left(\frac{i u}{4 \alpha}\right)^{m} \\
& \times\left[e^{-i \alpha} I_{m}(u)-e^{i \alpha} J_{m}(u)\right] \frac{d^{m+n-1}}{d p^{m+n-1}}\left[\left.\frac{1}{p^{n}} \exp \left(\frac{-i u^{2}}{8 \alpha p}\right)\right|_{p=1}\right.
\end{aligned}
$$

In Appendix B, there were some typographical errors in Eqs. (B4), (B6), (B8), and (B9). These equations should read

$$
\begin{aligned}
& \vartheta_{n}=2 \pi(-1)^{n}\left(t-z^{\prime} / v\right)^{2 n-1} \operatorname{Res}_{\theta=-i \alpha}\left\{\frac{1}{\left(\theta^{2}+\alpha^{2}\right)^{n}} \exp \left[\theta-\frac{u^{2}}{4(\theta-i \alpha)}+\frac{u^{2}}{4(\theta+i \alpha)}\right]\right\} \\
& +2 \pi(-1)^{n}\left(t-z^{\prime} / v\right)^{2 n-1} \operatorname{Res}_{\theta=i \alpha}\left\{\frac{1}{\left(\theta^{2}+\alpha^{2}\right)^{n}} \exp \left[\theta-\frac{u^{2}}{4(\theta-i \alpha)}+\frac{u^{2}}{4(\theta+i \alpha)}\right]\right\}, \\
& \frac{1}{\left(\theta^{2}+\alpha^{2}\right)^{n}} \exp \left[\theta-\frac{u^{2}}{4(\theta-i \alpha)}+\frac{u^{2}}{4(\theta+i \alpha)}\right]=\frac{e^{-i \alpha}}{s^{n}(s-2 i \alpha)^{n}} \exp \left[\frac{-u^{2}}{4(s-2 i \alpha)}\right] \sum_{m=-\infty}^{\infty}\left(\frac{2 s}{u}\right)^{m} I_{m}(u) \\
& \operatorname{Res}_{\theta=-i \alpha}\left\{\frac{1}{\left(\theta^{2}+\alpha^{2}\right)^{n}} \exp \left[\theta-\frac{u^{2}}{4(\theta-i \alpha)}+\frac{u^{2}}{4(\theta+i \alpha)}\right]\right\}=e^{-i \alpha} \sum_{m=-\infty}^{n-1}\left(\frac{2}{u}\right)^{m} I_{m}(u) \operatorname{Res}_{s=0}\left\{\frac{s^{m-n}}{(s-2 i \alpha)^{n}} \exp \left[\frac{-u^{2}}{4(s-2 i \alpha)}\right]\right\} \\
& =-e^{-i \alpha} \sum_{m=1-n}^{\infty}\left(\frac{-u}{2}\right)^{m} \frac{I_{m}(u)}{(m+n-1) !} \\
& \times \frac{d^{m+n-1}}{d r^{m+n-1}}\left\{\left.\frac{1}{(r+2 i \alpha)^{n}} \exp \left[\frac{u^{2}}{4(r+2 i \alpha)}\right]\right|_{r=0},\right.
\end{aligned}
$$

and

$$
\begin{aligned}
\operatorname{Res}_{\theta=i \alpha}\left\{\frac{1}{\left(\theta^{2}+\alpha^{2}\right)^{n}} \exp \left[\theta-\frac{u^{2}}{4(\theta-i \alpha)}+\frac{u^{2}}{4(\theta+i \alpha)}\right]\right\}= & e^{i \alpha} \sum_{m=1-n}^{\infty}\left(\frac{-u}{2}\right)^{m} \frac{J_{m}(u)}{(m+n-1) !} \\
& \times \frac{d^{m+n-1}}{d r^{m+n-1}}\left\{\left.\frac{1}{(r+2 i \alpha)^{n}} \exp \left[\frac{u^{2}}{4(r+2 i \alpha)}\right]\right|_{r=0} .\right.
\end{aligned}
$$

Finally, we add the following equation to the end of Appendix B:

$$
\vartheta_{1} \approx \frac{-i \pi e^{-i J\left(z^{\prime}\right) / 4}}{\omega_{\beta}}\left\{e^{-i \alpha}\left[I_{0}(u)-\frac{i u}{4 \alpha} I_{1}(u)\right]-e^{i \alpha}\left[J_{0}(u)-\frac{i u}{4 \alpha} J_{1}(u)\right]\right\} .
$$

\section{ACKNOWLEDGMENTS}

We would like to thank Dr. D. M. Pestrikov for bringing to our attention that if the equal sign were used in Eqs. (34), (36), (38), and (41) then zero would be the only solution for $\hat{Y}$. This work was supported by the U.S. Department of Energy.

[1] T. F. Wang, P. J. Channell, R. J. Macek, and R. C. Davidson, Phys. Rev. ST Accel. Beams 6, 014204 (2003). 